\title{
On the space of morphisms into generic real algebraic varieties
}

\author{
RICCARDO GHILONI
}

\begin{abstract}
We introduce a notion of generic real algebraic variety and we study the space of morphisms into these varieties. Let $Z$ be a real algebraic variety. We say that $Z$ is generic if there exist a finite family $\left\{D_{i}\right\}_{i=1}^{n}$ of irreducible real algebraic curves with genus $\geq 2$ and a biregular embedding of $Z$ into the product variety $\prod_{i=1}^{n} D_{i}$. A bijective map $\varphi: \widetilde{Z} \rightarrow Z$ from a real algebraic variety $\tilde{Z}$ to $Z$ is called weak change of the algebraic structure of $Z$ if it is regular and its inverse is a Nash map. Generic real algebraic varieties are "generic" in the sense specified by the following result: For each real algebraic variety $Z$ and for integer $k$, there exists an algebraic family $\left\{\varphi_{t}: \widetilde{Z}_{t} \rightarrow Z\right\}_{t \in \mathbb{R}^{k}}$ of weak changes of the algebraic structure of $Z$ such that $\widetilde{Z}_{0}=Z, \varphi_{0}$ is the identity map on $Z$ and, for each $t \in \mathbb{R}^{k} \backslash\{0\}, \widetilde{Z}_{t}$ is generic. Let $X$ and $Y$ be nonsingular irreducible real algebraic varieties. Regard the set $\mathcal{R}(X, Y)$ of regular maps from $X$ to $Y$ as a subspace of the corresponding set $\mathcal{N}(X, Y)$ of Nash maps, equipped with the $C^{\infty}$ compact-open topology. We prove that, if $Y$ is generic, then $\mathcal{R}(X, Y)$ is closed and nowhere dense in $\mathcal{N}(X, Y)$, and has a semi-algebraic structure. Moreover, the set of dominating regular maps from $X$ to $Y$ is finite. A version of the preceding results in which $X$ and $Y$ can be singular is given also.
\end{abstract}

Mathematics Subject Classification (2000): 14P05 (primary); 14P20 (secondary).

\section{Introduction and main theorems}

The aim of this paper is to study the topology of the space $\mathcal{R}$ of morphisms between real algebraic varieties $X$ and $Y$, and to prove the finiteness of the set $\mathcal{D}$ of dominating morphisms from $X$ to $Y$, when the target space $Y$ is generic in a suitable sense. Our main idea is to reduce to the 1-dimensional case in which, via complexification, it is possible to use classical results relating the genus of complex algebraic curves and morphisms between them. This strategy leads to two invariants generalizing the genus of an irreducible real algebraic curve, called curve genus and toric genus.

Received July 19, 2004; accepted in revised form August 3, 2006. 
A real algebraic variety is said to be generic if its toric genus is $\geq 2$. We will show that, given any real algebraic variety $X$, there exist algebraic families of generic real algebraic varieties arbitrarily close to $X$ both in an algebraic sense and in a topological sense. We will prove that, when $Y$ is generic, $\mathcal{R}$ is topologically small in the set of continuous semi-algebraic maps from $X$ to $Y$ (or Nash maps if $X$ and $Y$ are nonsingular) equipped with a suitable topology and it has a semi-algebraic structure. Moreover, $\mathcal{D}$ is finite.

In what follows, by a real algebraic variety, we mean a positive dimensional irreducible algebraic subset of some $\mathbb{R}^{n}$. Regular maps and algebraic subvarieties are understood in the sense of Serre [25, 4]. Moreover, algebraic subvarieties are assumed to be irreducible and Zariski closed. By a real algebraic curve, we mean a 1-dimensional real algebraic variety. Unless otherwise indicated, all real algebraic varieties we consider are equipped with the strong topology induced by the ordering structure on $\mathbb{R}$. We will use standard notions from real semi-algebraic geometry also (see [4]).

Let $X \subset \mathbb{R}^{n}$ be a real algebraic variety. We indicate by Nonsing $(X)$ the set of nonsingular points of $X$. If $X=\operatorname{Nonsing}(X)$, then $X$ is called nonsingular. Let $Y \subset \mathbb{R}^{m}$ be another real algebraic variety. Indicate by $\mathcal{R}(X, Y)$ the set of regular maps from $X$ to $Y$ and by $\operatorname{Dom}(X, Y)$ the set of all $f \in \mathcal{R}(X, Y)$ such that $f$ is dominating, i.e., $f(X)$ is Zariski dense in $Y$. A map $f: X \rightarrow Y$ is said to be Nash if there exist an open semi-algebraic neighborhood $V$ of $X$ in $\mathbb{R}^{n}$ and an extension $F: V \rightarrow \mathbb{R}^{m}$ of $f$ from $V$ to $\mathbb{R}^{m}$, which is Nash, i.e., semi-algebraic of class $C^{\infty}$. Indicate by $\mathcal{N}(X, Y)$ the set of Nash maps from $X$ to $Y$ and by $\mathcal{S}^{0}(X, Y)$ the set of continuous semi-algebraic maps from $X$ to $Y$.

Before presenting our invariants and related results, we need to recall some well-known facts about morphisms between real algebraic curves.

Equip each projective space $\mathbb{P}^{n}(\mathbb{C})$ with its natural structure of complex algebraic variety, indicate by $\sigma_{n}: \mathbb{P}^{n}(\mathbb{C}) \rightarrow \mathbb{P}^{n}(\mathbb{C})$ the complex conjugation map and identify $\mathbb{P}^{n}(\mathbb{R})$ with the fixed point set of $\sigma_{n}$. A subset $S$ of $\mathbb{P}^{n}(\mathbb{C})$ is said to be defined over $\mathbb{R}$ if it is $\sigma_{n}$-invariant and its real part $S(\mathbb{R})$ is defined as the intersection $S \cap \mathbb{P}^{n}(\mathbb{R})$. By a complex algebraic curve (defined over $\mathbb{R}$ ), we mean a 1-dimensional nonsingular irreducible Zariski closed algebraic subvariety of some $\mathbb{P}^{n}(\mathbb{C})$ (defined over $\mathbb{R}$ ). For each real algebraic curve $D$, there exists a unique (up to complex biregular isomorphism) complex algebraic curve $D_{\mathbb{C}}$ defined over $\mathbb{R}$ such that $D_{\mathbb{C}}(\mathbb{R})$, viewed in the natural way as a real algebraic variety, is birationally equivalent to $D$. Such a curve $D_{\mathbb{C}}$ is called nonsingular projective complexification of $D$. The genus $g(D)$ of $D$ is defined to be the genus $g\left(D_{\mathbb{C}}\right)$ of $D_{\mathbb{C}}$.

Let $D$ and $E$ be real algebraic curves, and let $D_{\mathbb{C}}$ and $E_{\mathbb{C}}$ be the nonsingular projective complexifications of $D$ and of $E$ respectively. $\operatorname{Suppose} \operatorname{Dom}(D, E)$ is non-empty and fix $f \in \operatorname{Dom}(D, E)$. Let $D_{f}:=\operatorname{Nonsing}(D) \cap f^{-1}(\operatorname{Nonsing}(E))$ and let $f^{\prime}: D_{f} \rightarrow \operatorname{Nonsing}(E)$ be the restriction of $f$ from $D_{f}$ to $\operatorname{Nonsing}(E)$. Identify $D_{f}$ and Nonsing $(E)$ with Zariski open subsets of $D_{\mathbb{C}}(\mathbb{R})$ and of $E_{\mathbb{C}}(\mathbb{R})$ respectively. By Zariski's Main Theorem, we know that there exists a unique complex regular map $f_{\mathbb{C}}: D_{\mathbb{C}} \rightarrow E_{\mathbb{C}}$ which extends $f^{\prime}$. This map is called complexification of $f$. Since $f$ is dominating, $f_{\mathbb{C}}$ is dominating also. In particular, Hurwitz's 
formula applies to $f_{\mathbb{C}}$. It follows at once that $g(D) \geq g(E)$. Furthermore, as observed by Bochnak and Kucharz in [5], if $g(E)=1$, then the restriction of $f$ to $D_{f}$ has at most $2 g(D)-2$ critical points. Suppose now $g(E)=g\left(E_{\mathbb{C}}\right) \geq 2$. Indicate by $\sharp S$ the cardinality of the set $S$. The finiteness theorem of de Franchis [11] asserts that the set $\operatorname{Dom}_{\mathbb{C}}\left(D_{\mathbb{C}}, E_{\mathbb{C}}\right)$ of dominating complex regular maps from $D_{\mathbb{C}}$ to $E_{\mathbb{C}}$ is finite. In [23], H. Martens improved this result showing first the existence of an upper bound for $\sharp \operatorname{Dom}_{\mathbb{C}}\left(D_{\mathbb{C}}, E_{\mathbb{C}}\right)$ depending only on $g(D)=g\left(D_{\mathbb{C}}\right)$. By complexification, we infer that $\operatorname{Dom}(D, E)$ is finite and there exists an upper bound for $\sharp \operatorname{Dom}(D, E)$ depending only on $g(D)$. Combining the preceding observations with standard Nash approximation results, we obtain immediately the following two theorems (see [5]).

Theorem $\mathcal{R}_{1}$. Let $D$ and $E$ be nonsingular real algebraic curves. Equip $\mathcal{N}(D, E)$ with the $C^{\infty}$ compact-open topology (the weak $C^{\infty}$ topology in the terminology used in [14]) and consider $\mathcal{R}(D, E)$ as a subspace of $\mathcal{N}(D, E)$. The following holds:

(1) Suppose $g(D)<g(E)$. Then every regular map from $D$ to $E$ is constant. In particular, $\mathcal{R}(D, E)$ is closed and nowhere dense in $\mathcal{N}(D, E)$, and is homeomorphic to $E$.

(2) Suppose $g(E) \geq 1$. Then $\mathcal{R}(D, E)$ is not dense in $\mathcal{N}(D, E)$.

(3) Suppose $g(E) \geq 2$. Then $\mathcal{R}(D, E)$ is closed and nowhere dense in $\mathcal{N}(D, E)$, and is homeomorphic to the disjoint topological sum of $E$ and a finite discrete space.

Theorem $\mathcal{D}_{1}$. Let $D$ and $E$ be real algebraic curves (not necessarily nonsingular). Suppose $g(E) \geq 2$. Then $\operatorname{Dom}(D, E)$ is finite and there exists an upper bound for its cardinality in terms of $g(D)$ and $g(E)$ only.

The remainder of this section is subdivided into three subsections. In Subsection 1.1, we define the curve genus and the toric genus, and we give some their properties and applications. The main result is Theorem 1.9. This theorem specifies in which sense it is possible to assert that nonsingular real algebraic varieties with arbitrarily high toric genus are generic. More precisely, given a nonsingular real algebraic variety $X$, for each pair of integers $k$ and $N$, we construct an algebraic family $\left\{\varphi_{t}: \widetilde{X}_{t} \rightarrow X\right\}_{t \in \mathbb{R}^{k}}$ of regular maps from nonsingular real algebraic varieties to $X$ parametrized by $\mathbb{R}^{k}$ such that each map $\varphi_{t}$ is a Nash isomorphism, $\widetilde{X}_{0}=X$, $\varphi_{0}$ is the identity on $X$ and, for each $t \in \mathbb{R}^{k} \backslash\{0\}$, the toric genus of $\widetilde{X}_{t}$ is $\geq N$. In Subsections 1.2 and 1.3 , we extend Theorems $\mathcal{R}_{1}$ and $\mathcal{D}_{1}$ to morphisms between real algebraic varieties $X$ and $Y$ of any dimension. In substance, the curve genus of $X$ and the toric genus of $Y$ will play the roles of $g(D)$ and of $g(E)$ respectively. In this way, calling generic a real algebraic variety with toric genus $\geq 2$, we infer the following theorem, which is the most significant result of this paper. 
Theorem $\mathcal{R}+\mathcal{D}$. Let $X$ and $Y$ be nonsingular real algebraic varieties. Suppose $Y$ generic. Equip $\mathcal{N}(X, Y)$ with the $C^{\infty}$ compact-open topology and consider $\mathcal{R}(X, Y)$ as a subspace of $\mathcal{N}(X, Y)$. Then $\mathcal{R}(X, Y)$ is closed and nowhere dense in $\mathcal{N}(X, Y)$, and admits a semi-algebraic structure. Furthermore, $\operatorname{Dom}(X, Y)$ is finite and there exists an upper bound for its cardinality in terms of the curve genus of $X$, the curve genus of $Y$ and the toric genus of $Y$ only.

Remark $\mathcal{R}+\mathcal{D}$. Let $X$ and $Y$ be as above. Suppose $X$ compact. The classical Stone-Weierstrass approximation theorem implies that $\mathcal{R}(X, Y)$ is dense in $\mathcal{N}(X, Y)$ (or, equivalently, in $C^{\infty}(X, Y)$ ), equipped with the $C^{\infty}$ compact-open topology, if $Y$ is equal to some $\mathbb{R}^{m}$. An important, but very difficult, problem is to try to extend this result to other target spaces $Y$. General results are known only when $Y$ is $S^{1}, S^{2}, S^{4}$, a grassmannian or, more generally, a rational real algebraic variety. The most part of these results follows from the deep connections existing between the notion of strongly algebraic vector bundle over $Y$ introduced in [3], the algebraic and topological $K$-theories of $Y$ and the algebraic properties of the $\mathbb{Z} / 2$-cohomology of $Y$. We refer the interested reader to Chapter 12, Section 13.3 and related references of [4], and to the papers [5, 6, 7, 8, 9, 16, 17, 18, 20, 21, 22]. Theorem $\mathcal{R}+\mathcal{D}$ gives a justification of the difficulty of this algebraic approximation problem: "given a generic target space, such a problem has a negative solution".

The proofs of all our results are given in Section 2.

In this paper, we work with the field $\mathbb{R}$ of real numbers only, but we shall deal with arbitrary real closed fields. We have to be careful regarding one point only: the notion of compactness. In the definitions of compact-open topology and of $C^{\infty}$ compact-open topology, one must replace "compact" with "closed and bounded" (see Subsection 2.4 at the end of the paper). The theorems presented here were announced in [12]. For further results concerning morphisms between real algebraic varieties, we refer the reader to [13].

We wish to thank the referee for his precious advice regarding the presentation of this paper. A special thank goes to E. Ballico, M. Shiota and A. Tognoli for several useful discussions.

\subsection{Curve genus and toric genus}

Let $X \subset \mathbb{R}^{n}$ be a real algebraic variety. If an algebraic subvariety $D$ of $X$ is a real algebraic curve, then we say that $D$ is a real algebraic curve of $X$. Let $\mathbb{N}$ be the set of all non-negative integers. For each $k \in \mathbb{N}$ and for each $x \in X$, we denote by $\mathcal{C}_{X}(k, x)$ the set of real algebraic curves of $X$ of genus $k$ and containing $x$. Let us introduce the notion of complete intersection degree of $X$ in $\mathbb{R}^{n}$, which gives a measure of the complexity of $X$. Let $r:=\operatorname{dim}(X)$. First, suppose $r<$ $n$. We define the complete intersection degree $\operatorname{cideg}\left(X, \mathbb{R}^{n}\right)$ of $X$ in $\mathbb{R}^{n}$ as the minimum integer $c$ such that there exist a point $p \in \operatorname{Nonsing}(X)$ and polynomials $P_{1}, \ldots, P_{n-r}$ in $\mathbb{R}\left[x_{1}, \ldots, x_{n}\right]$ vanishing on $X$ with independent gradients at $p$ and $c=\prod_{i=1}^{n-r} \operatorname{deg}\left(P_{i}\right)$. If $r=n$, then we consider $\operatorname{cideg}\left(X, \mathbb{R}^{n}\right)$ equal to 1 . 
Lemma 1.1. Let $X \subset \mathbb{R}^{n}$ be a real algebraic variety, let $c:=\operatorname{cideg}\left(X, \mathbb{R}^{n}\right)$ and let $e=(c-1)(c-2) / 2$. Then, for each $x \in \operatorname{Nonsing}(X)$, there exists $k \in\{0,1, \ldots, e\}$ (depending on $x$ ) such that $\bigcup_{D \in \mathcal{C}_{X}(k, x)} D$ is Zariski dense in $X$.

This result allows to give the following definition.

Definition 1.2. Let $X$ be a real algebraic variety. We define the curve genus $p_{c}(X)$ of $X$ as follows:

$$
p_{c}(X):=\min _{x \in \operatorname{Nonsing}(X)} \min \left\{k \in \mathbb{N} \mid \bigcup_{D \in \mathcal{C}_{X}(k, x)} D \text { is Zariski dense in } X\right\} .
$$

From Lemma 1.1, we infer (see Remark 2.1 also):

Corollary 1.3. Let $X \subset \mathbb{R}^{n}$ be a real algebraic variety and let $c:=\operatorname{cideg}\left(X, \mathbb{R}^{n}\right)$. Then it holds: $p_{c}(X) \leq(c-1)(c-2) / 2$.

Let $X \subset \mathbb{R}^{n}$ be a real algebraic variety. Recall that a map $\psi: X \rightarrow Y$ from $X$ to a real algebraic variety $Y$ is said to be a biregular embedding of $X$ into $Y$ if $\psi(X)$ is an algebraic subvariety of $Y$ and the restriction of $\psi$ from $X$ to $\psi(X)$ is a biregular isomorphism. We denote by $\mathcal{T}(X)$ the set of all integers $h \in \mathbb{N}$ such that there exist a finite family $\left\{D_{i}\right\}_{i=1}^{m}$ of real algebraic curves with $\min _{i \in\{1, \ldots, m\}} g\left(D_{i}\right)=h$ and a biregular embedding of $X$ into the product variety $\prod_{i=1}^{m} D_{i}$. Since $X$ is contained in $\mathbb{R}^{n}$ and $\mathbb{R}$ is a real algebraic curve of genus 0 , the set $\mathcal{T}(X)$ contains 0 and hence it is non-empty.

Definition 1.4. Let $X$ be a real algebraic variety. We define the toric genus $p_{t}(X)$ of $X$ by $p_{t}(X):=\sup \mathcal{T}(X)$.

Lemma 1.5. Let $X$ be a real algebraic variety. Then the toric genus $p_{t}(X)$ of $X$ is finite and it holds: $p_{t}(X) \leq p_{c}(X)$.

The curve and toric genera are biregular invariants on real algebraic varieties. If $X$ is a real algebraic curve, then $p_{c}(X)=p_{t}(X)=g(X)$.

Example 1.6. (i) Let $X$ be a rational real algebraic variety, i.e., a nonsingular real algebraic variety birationally equivalent to some $\mathbb{R}^{n}$. The definition of curve genus and the preceding lemma imply that $p_{c}(X)=p_{t}(X)=0$.

(ii) Let $\left\{D_{i}\right\}_{i \in I}$ be a finite family of real algebraic curves and let $T$ be the product variety $\prod_{i \in I} D_{i}$. Define $a:=\min _{i \in I} g\left(D_{i}\right)$ and $b:=\max _{i \in I} g\left(D_{i}\right)$. By a simple argument based on Hurwitz's formula (see the proof of Lemma 1.5), we obtain that $p_{c}(T) \geq b$ and $p_{t}(T)=a$. It follows that, for each integer $n \geq$ 2 , there exist real algebraic varieties $T$ of dimension $n$ for which the difference $p_{c}(T)-p_{t}(T)$ is arbitrarily large (see Remark 2.9 also).

We give now a rigorous meaning to the following rough assertion: "Given a nonsingular real algebraic variety $X$ and an integer $N$, there exist many nonsingular real algebraic varieties arbitrarily close to $X$ whose toric genus is $\geq N$ ".

Definition 1.7. Let $X$ and $\widetilde{X}$ be real algebraic varieties. We say that a bijective map $\varphi: \widetilde{X} \rightarrow X$ is a weak change of the algebraic structure of $X$ if it is regular and $\varphi^{-1}$ is a Nash map. 
Observe that, if $\varphi: \widetilde{X} \rightarrow X$ has the above properties, then $\varphi^{-1}$ maps nonsingular points into nonsingular points. In particular, if $X$ is nonsingular, then $\widetilde{X}$ is nonsingular also.

Definition 1.8. Let $X, Z$ and $X^{*}$ be real algebraic varieties, let $z_{0} \in Z$ and let $\pi: X^{*} \rightarrow Z$ be a regular map. A map $\xi: X^{*} \rightarrow X$ is called weak deformation of $X$ parametrized by $\left(\pi, z_{0}\right)$ if it is regular, the restriction of $\xi$ to $\pi^{-1}\left(z_{0}\right)$ is a biregular isomorphism and, for each $z \in Z \backslash\left\{z_{0}\right\}$, the restriction of $\xi$ to $\pi^{-1}(z)$ is a weak change of the algebraic structure of $X$.

Let $M$ be an affine Nash manifold and let $k \in \mathbb{N}$. We say that a Nash map $F: M \times \mathbb{R}^{k} \rightarrow M$ is a Nash $k$-diffeotopy of $M$ if, for each $t \in \mathbb{R}^{k}$, the restriction $F_{t}$ of $F$ to $M \times\{t\}$ is a Nash isomorphism and $F_{0}$ is the identity map $i d_{M}$ on $M$. Equip $\mathcal{N}(M, M)$ with the $C^{\infty}$ compact-open topology and let $\mathcal{U}$ be a neighborhood of $i d_{M}$ in $\mathcal{N}(M, M)$. We say that $F$ is in $\mathcal{U}$ if $F_{t} \in \mathcal{U}$ for each $t \in \mathbb{R}^{k}$. In what follows, for each $m>n$, we identify $\mathbb{R}^{n} \times \mathbb{R}^{m-n}$ with $\mathbb{R}^{m}$ and $\mathbb{R}^{n}$ with the subspace $\mathbb{R}^{n} \times\{0\}$ of $\mathbb{R}^{m}$.

Theorem 1.9. Let $X \subset \mathbb{R}^{n}$ be a real algebraic variety and let $k, N \in \mathbb{N}$. Suppose $X$ nonsingular. Denote by $\pi: \mathbb{R}^{2 n} \times \mathbb{R}^{k} \rightarrow \mathbb{R}^{k}$ the natural projection and by 0 the origin of $\mathbb{R}^{k}$. Choose a neighborhood $\mathcal{U}$ of id $\mathbb{R}^{2 n}$ in $\mathcal{N}\left(\mathbb{R}^{2 n}, \mathbb{R}^{2 n}\right)$ with respect to the $C^{\infty}$ compact-open topology. Then there exist a real algebraic variety $X^{*} \subset$ $\mathbb{R}^{2 n} \times \mathbb{R}^{k}$ and a weak deformation $\xi: X^{*} \rightarrow X$ of $X$ parametrized by $\left(\left.\pi\right|_{\left.X^{*}, 0\right)}\right.$ such that:

(1) $X^{*} \cap \pi^{-1}(0)=X$ and $\xi$ extends to a Nash $k$-diffeotopy of $\mathbb{R}^{2 n}$ in $\mathcal{U}$.

(2) For each $t \in \mathbb{R}^{k} \backslash\{0\}$, the toric genus of $X^{*} \cap \pi^{-1}(t)$ is $\geq N$.

Remark 1.10. (i) We conjecture that, in the statement of Theorem 1.9, it is possible to add the following point: (3) For each $t, s \in \mathbb{R}^{k}$ with $t \neq s, X^{*} \cap \pi^{-1}(t)$ and $X^{*} \cap \pi^{-1}(s)$ are birationally nonequivalent.

(ii) Our invariants can be defined as well in the complex setting. However, the complex toric genus does not seem to be a significant notion. A reason is that the preceding theorem cannot be extended to the complex case: for example, it is wellknown that the complex projective line $\mathbb{P}^{1}(\mathbb{C})$ has a unique structure of nonsingular complex algebraic variety.

Theorem 1.9 contains the following result.

Corollary 1.11. Let $X$ be a nonsingular real algebraic variety and let $N \in \mathbb{N}$. Then there exists a weak change $\varphi: \widetilde{X} \rightarrow X$ of the algebraic structure of $X$ such that $p_{t}(\tilde{X}) \geq N$.

In the remainder of this subsection, we give some applications of the notions and of the results presented above.

Theorem 1.12. Let $X$ and $Y$ be real algebraic varieties. If $p_{c}(X)<p_{t}(Y)$, then every regular map from $X$ to $Y$ is constant. 
By combining this result with Corollary 1.11, we obtain:

Corollary 1.13. Let $Y$ be a nonsingular real algebraic variety and let $\left\{X_{i}\right\}_{i \in I}$ be a family of real algebraic varieties such that $\sup _{i \in I} p_{c}\left(X_{i}\right)$ is finite (for example, the family of rational real algebraic varieties). Then there exists a weak change $\varphi: \widetilde{Y} \rightarrow Y$ of the algebraic structure of $Y$ such that, for each $i \in I$, every regular map from $X_{i}$ to $\widetilde{Y}$ is constant.

Remark 1.14. Let $Y$ be a nonsingular real algebraic variety. Recall that the homology of $Y$ is called totally algebraic if each $\mathbb{Z} / 2$-homology class of $Y$ can be represented by a Zariski closed subset of $Y$ (see Chapters 11-14 of [4]). We say that the homotopy of $Y$ is algebraically trivial if, for each integer $n \in \mathbb{N} \backslash\{0\}$, every regular map from the standard $n$-sphere $S^{n}$ to $Y$ is homotopic to a constant. For each $\underset{\sim}{n} \in \mathbb{N} \backslash\{0\}, S^{n}$ is rational so, by Corollary 1.13 , there exists a weak change $\varphi: \widetilde{Y} \rightarrow Y$ of the algebraic structure of $Y$ such that the homotopy of $\widetilde{Y}$ is algebraically trivial. Evidently, if the homology of $Y$ is totally algebraic, then the homology of $\widetilde{Y}$ is totally algebraic also. As an immediate consequence of these facts, we infer that every connected compact smooth manifold of dimension $\leq 5$ is diffeomorphic to a nonsingular real algebraic variety with totally algebraic homology whose homotopy is algebraically trivial (see [4, Theorem 11.3.12]).

Remark 1.15. Theorem 1.9 has the following singular version: "Let $X \subset \mathbb{R}^{n}$ be a real algebraic set (not necessarily irreducible, nonsingular and of positive dimension) and let $k, N, \pi$ and $\mathcal{U}$ be as in the statement of the mentioned theorem. Then there exist an algebraic subset $X^{*}$ of $\mathbb{R}^{2 n} \times \mathbb{R}^{k}$ and a regular map $\xi: X^{*} \rightarrow X$ such that: (1) $X^{*} \cap \pi^{-1}(0)=X$ and $\xi$ extends to a Nash $k$-diffeotopy of $\mathbb{R}^{2 n}$ in $\mathcal{U}$. (2) For each $t \in \mathbb{R}^{k} \backslash\{0\}, X^{*} \cap \pi^{-1}(t)$ can be biregularly embedded into a finite product of real algebraic curves of genus $\geq N$ ". This result and Theorem 1.12 imply the following version of Corollary 1.13: "Let $Y$ be a real algebraic set (not necessarily irreducible, nonsingular and of positive dimension) and let $\left\{X_{i}\right\}_{i \in I}$ be as in the statement of the mentioned corollary. Then there exist a real algebraic set $\widetilde{Y}$ and a regular map $\varphi: \widetilde{Y} \rightarrow Y$ such that $\varphi$ is a Nash isomorphism and, for each $i \in I$, every regular map from $X_{i}$ to $\widetilde{Y}$ is constant".

\subsection{Topology of morphism space}

Let $T$ be a topological space and let $S$ be a subset of $T$. Recall that $S$ is said to be nowhere dense in $T$ if the interior of the closure of $S$ in $T$ is empty. Indicate by Is $(T)$ the set of isolated points of $T$. We say that $S$ is nowhere dense up to isolated points in $T$ if $S \backslash \operatorname{Is}(T)$ is nowhere dense in $T$. Observe that, if $T$ is a real algebraic variety, then Is $(T)$ may be non-empty. On the contrary, if $T$ is a nonsingular real algebraic variety, then Is $(T)$ is empty.

Let $X$ and $Y$ be fixed real algebraic varieties. We have:

Theorem 1.16. Equip $\mathcal{S}^{0}(X, Y)$ with the compact-open topology and consider $\mathcal{R}(X, Y)$ as a subspace of $\mathcal{S}^{0}(X, Y)$. The following holds: 
(0) $\operatorname{Is}\left(\mathcal{S}^{0}(X, Y)\right)=\left\{f \in \mathcal{S}^{0}(X, Y) \mid f(X) \subset \operatorname{Is}(Y)\right\}$ so $\operatorname{Is}\left(\mathcal{S}^{0}(X, Y)\right)$ is finite and $\mathcal{R}(X, Y) \cap \operatorname{Is}\left(\mathcal{S}^{0}(X, Y)\right)$ is equal to the set of all constant maps from $X$ to $Y$ whose image is contained in $\operatorname{Is}(Y)$.

(1) Suppose $p_{c}(X)<p_{t}(Y)$. Then every regular map from $X$ to $Y$ is constant. In particular, $\mathcal{R}(X, Y)$ is closed and nowhere dense up to isolated points in $\mathcal{S}^{0}(X, Y)$, and is homeomorphic to $Y$.

(2) Suppose $p_{t}(Y) \geq 1$. Then $\mathcal{R}(X, Y)$ is nowhere dense up to isolated points in $\mathcal{S}^{0}(X, Y)$.

(3) Suppose $p_{t}(Y) \geq 2$. Then $\mathcal{R}(X, Y)$ is closed in $\mathcal{S}^{0}(X, Y)$ and is homeomorphic to a closed semi-algebraic set. Furthermore, such a semi-algebraic set can be choosen bounded if $Y$ is bounded.

Suppose now $X$ and $Y$ nonsingular. We have:

Theorem 1.17. Equip $\mathcal{N}(X, Y)$ with the $C^{\infty}$ compact-open topology and consider $\mathcal{R}(X, Y)$ as a subspace of $\mathcal{N}(X, Y)$. The following holds:

(1) Suppose $p_{c}(X)<p_{t}(Y)$. Then every regular map from $X$ to $Y$ is constant. In particular, $\mathcal{R}(X, Y)$ is closed and nowhere dense in $\mathcal{N}(X, Y)$, and is homeomorphic to $Y$.

(2) Suppose $p_{t}(Y) \geq 1$. Then the interior of the closure of $\mathcal{R}(X, Y)$ in $\mathcal{N}(X, Y)$ is contained in the set of all maps in $\mathcal{N}(X, Y)$ whose fibers are finite. In particular, $\mathcal{R}(X, Y)$ is not dense in $\mathcal{N}(X, Y)$ and, when $\operatorname{dim}(X)>\operatorname{dim}(Y), \mathcal{R}(X, Y)$ is nowhere dense in $\mathcal{N}(X, Y)$.

(3) Suppose $p_{t}(Y) \geq 2$. Then $\mathcal{R}(X, Y)$ is closed and nowhere dense in $\mathcal{N}(X, Y)$, and is homeomorphic to a closed semi-algebraic set. Furthermore, such a semi-algebraic set can be choosen bounded if $Y$ is bounded.

\subsection{Finiteness of dominating morphism space}

We need some preparations. Let $A$ be a complex algebraic curve. By the de Franchis-Martens finiteness theorem, we know that the set $\operatorname{Dom}_{\mathbb{C}}(A, B)$ of dominating complex regular maps from $A$ to a fixed complex algebraic curve $B$ with genus $\geq 2$ is finite and there exists an upper bound for $\sharp \operatorname{Dom}_{\mathbb{C}}(A, B)$ in terms of $g(A)$ only. Indicate by $\mathcal{M}: \mathbb{N} \times(\mathbb{N} \backslash\{0,1\}) \rightarrow \mathbb{N}$ the function which maps $(a, b) \in \mathbb{N} \times(\mathbb{N} \backslash\{0,1\})$ into the maximum integer $k \in \mathbb{N}$ such that there exist complex algebraic curves $A$ and $B$ with $g(A)=a, g(B)=b$ and $\sharp \operatorname{Dom}_{\mathbb{C}}(A, B)=k$. By Hurwitz's formula, it follows that $\mathcal{M}(a, b)=0$ if $a<b$ so we can define the function $\mathcal{M}_{*}$ as the smallest function $f: \mathbb{N} \times(\mathbb{N} \backslash\{0,1\}) \rightarrow \mathbb{N}$ such that $\mathcal{M} \leq f$ and $f(a, b+1) \leq f(a, b) \leq f(a+1, b)$ for each $(a, b) \in \mathbb{N} \times(\mathbb{N} \backslash\{0,1\})$. Explicit upper bounds for $\mathcal{M}_{*}$ can be found in [24, 28].

Let us recall another finiteness result of complex algebraic geometry. Let $G_{\mathbb{C}}(A)$ be the set of dominating regular maps from $A$ to some complex algebraic curve $B$ with genus $\geq 2$. Define an equivalence relation $\mathcal{H}_{\mathbb{C}}$ on $G_{\mathbb{C}}(A)$ as follows:

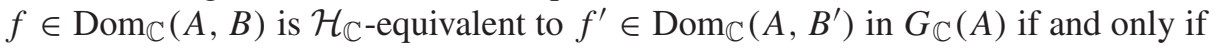
there exists a complex biregular isomorphism $h: B \rightarrow B^{\prime}$ such that $f^{\prime}=h \circ f$. 
Indicate by $\mathcal{G}_{\mathbb{C}}(A)$ the set of $\mathcal{H}_{\mathbb{C}}$-equivalence classes of $G_{\mathbb{C}}(A)$. The de FranchisSeveri theorem $[11,26]$ asserts that $\mathcal{G}_{\mathbb{C}}(A)$ is finite. In [15], Howard and Sommese proved that there exists an upper bound for $\sharp \mathcal{G}_{\mathbb{C}}(A)$ in terms of $g(A)$ only. Indicate by $\mathcal{F}: \mathbb{N} \backslash\{0,1\} \rightarrow \mathbb{N}$ the function which maps $a \in \mathbb{N} \backslash\{0,1\}$ into the maximum integer $k \in \mathbb{N}$ such that there exists a complex algebraic curve $A$ with $g(A)=a$ and $\sharp \mathcal{G}_{\mathbb{C}}(A)=k$. Observe that, by Hurwitz's formula, we have that $\mathcal{F}(2)=1$. Explicit upper bounds for $\mathcal{F}$ can be found in $[1,15,19]$.

We can now present two extensions of Theorem $\mathcal{D}_{1}$.

Theorem 1.18. Let $X$ and $Y$ be real algebraic varieties. If $p_{t}(Y) \geq 2$, then $\operatorname{Dom}(X, Y)$ is finite and there exists an upper bound for its cardinality in terms of $p_{c}(X), p_{c}(Y)$ and $p_{t}(Y)$ only. More precisely, it holds:

$$
\sharp \operatorname{Dom}(X, Y) \leq \mathcal{M}_{*}\left(p_{c}(X), p_{t}(Y)\right)^{\mathcal{F}\left(p_{c}(Y)\right)} .
$$

Let $X$ and $Y$ be real algebraic varieties and let $f \in \mathcal{R}(X, Y)$. We say that $f$ is weakly dominating if the interior of the closure of $f(\operatorname{Nonsing}(X))$ in $Y$ is nonempty. Indicate by $w \operatorname{Dom}(X, Y)$ the set of weakly dominating regular maps from $X$ to $Y$. It is easy to see that if $f$ is dominating or is open in the usual sense, then it is weakly dominating also. However, in general, the converse is false. For example, if $X$ is the line $\mathbb{R}$ and $Y$ is the Whitney umbrella $\left\{(x, y, z) \in \mathbb{R}^{3} \mid y^{2}-z x^{2}=0\right\}$, then the regular map, which sends $x$ into $(0,0, x)$, is weakly dominating, but it is neither dominating nor open.

Theorem 1.19. Let $X$ and $Y$ be real algebraic varieties. If $p_{t}(Y) \geq 2$, then $\operatorname{wDom}(X, Y)$ is finite. In particular, there are only finitely many open regular maps from $X$ to $Y$.

\section{Proofs}

In this section, we will use some techniques and arguments contained in [13]. Since the latter paper has not yet appeared in print, we repeat them here.

\subsection{Results of Subsection 1.1}

Proof of Lemma 1.1. Fix $x \in \operatorname{Nonsing}(X)$. Define $r:=\operatorname{dim}(X)$. If $r=n$, then the union of all lines of $\mathbb{R}^{n}$ containing $x$ is equal to $\mathbb{R}^{n}=X$ so $\bigcup_{D \in \mathcal{C}_{X}(0, x)} D=X$ and the lemma is proved. Suppose $r<n$. By using an affine automorphism of $\mathbb{R}^{n}$ if needed, we may suppose that $x$ coincides with the origin of $\mathbb{R}^{n}$ and the tangent space of $X$ at $x$ is equal to the subspace $\mathbb{R}^{r} \times\{0\}$ of $\mathbb{R}^{n}=\mathbb{R}^{r} \times \mathbb{R}^{n-r}$. Applying the Implicit Function Theorem for Nash maps, we obtain an open ball $B_{r}(0,2 \varepsilon)$ of $\mathbb{R}^{r}$ centered at 0 with radious $2 \varepsilon>0$, an open semi-algebraic neighborhood $V$ of 0 in $\mathbb{R}^{n-r}$ and a Nash map $f: B_{r}(0,2 \varepsilon) \rightarrow V$ such that $X \cap\left(B_{r}(0,2 \varepsilon) \times V\right)$ is equal to the graph of $f$. Indicate by $\pi: \mathbb{R}^{r} \times \mathbb{R}^{n-r} \rightarrow \mathbb{R}^{r}$ the natural projection. Let $P_{1}, \ldots, P_{n-r}$ be polynomials in $\mathbb{R}\left[x_{1}, \ldots, x_{n}\right]$ vanishing on $X$ with 
independent gradients at some $q \in \operatorname{Nonsing}(X)$ such that $\prod_{i=1}^{n-r} \operatorname{deg}\left(P_{i}\right)=c$ and let $S$ be the Zariski closed subset of $X$ formed by all points $p \in X$ such that $\nabla P_{1}(p), \ldots, \nabla P_{n-r}(p)$ are dependent in $\mathbb{R}^{n}$. Since $q \notin S$ and $X$ is irreducible, it follows that $\operatorname{dim}(S)<r$. In particular, the Zariski closure $S^{*}$ of $\pi(S)$ in $\mathbb{R}^{r}$ has dimension $<r$ also. Let $W$ be a non-empty open semi-algebraic subset of $B_{r}(0,2 \varepsilon) \backslash\left(S^{*} \cup\{0\}\right)$. For each $w \in W$, let $N_{w}$ be the $(n-r+1)$-dimensional vector subspace of $\mathbb{R}^{n}$ generated by $\{(w, 0)\} \cup\left(\{0\} \times \mathbb{R}^{n-r}\right)$ and let $D_{w}$ be the irreducible component of $X \cap N_{w}$ containing 0 . Observe that, for each $w \in W$, $D_{w}$ is a real algebraic curve of $X$ containing $\{0,(w, f(w))\}$. We will show that, for each $w \in W, g\left(D_{w}\right) \leq e$. Let $w \in W$. For each $i \in\{1, \ldots, n-r\}$, define $X_{i}:=P_{i}^{-1}(0)$. Identify $\mathbb{R}^{n}$ with a subset of $\mathbb{P}^{n}(\mathbb{R})$ by the affine chart which maps $\left(x_{1}, \ldots, x_{n}\right)$ into $\left[x_{1}, \ldots, x_{n}, 1\right]$. Consider $X$ and each $X_{i}$ as subsets of $\mathbb{P}^{n}(\mathbb{R})$ and hence as subsets of $\mathbb{P}^{n}(\mathbb{C})$. Indicate by $D_{w, \mathbb{C}}, N_{w, \mathbb{C}}, X_{1, \mathbb{C}}, \ldots, X_{n-r, \mathbb{C}}$ the Zariski closures of $D_{w}, N_{w}, X_{1}, \ldots, X_{n-r}$ in $\mathbb{P}^{n}(\mathbb{C})$ respectively. Observe that $D_{w, \mathbb{C}}$ is an irreducible component of $N_{w, \mathbb{C}} \cap \bigcap_{i=1}^{n-r} X_{i, \mathbb{C}},(w, f(w)) \in N_{w, \mathbb{C}} \cap$ $\bigcap_{i=1}^{n-r} \operatorname{Nonsing}\left(X_{i, \mathbb{C}}\right)$ and $N_{w, \mathbb{C}}$ and $\left\{\operatorname{Nonsing}\left(X_{i, \mathbb{C}}\right)\right\}_{i=1}^{n-r}$ are in general position in $\mathbb{P}^{n}(\mathbb{C})$ locally at $(w, f(w))$. Under these conditions, Bezout's theorem implies that $\operatorname{deg}\left(D_{w, \mathbb{C}}\right) \leq \prod_{i=1}^{n-r} \operatorname{deg}\left(X_{i, \mathbb{C}}\right) \leq c$. By the Castelnuovo Bound Theorem (see the following remark), we obtain that the geometric genus of $D_{w, \mathbb{C}}$, which is equal to $g\left(D_{w}\right)$, is $\leq e$ as desired. Let us complete the proof. The set $\bigcup_{w \in W} D_{w}$ contains $\{(w, f(w)) \in \operatorname{Nonsing}(X) \mid w \in W\}$ so it is Zariski dense in $X$. Since $X$ is irreducible and $\bigcup_{w \in W} D_{w} \subset \bigcup_{k=0}^{e} \bigcup_{D \in \mathcal{C}_{X}(k, x)} D$, there exists $k \in\{0,1, \ldots, e\}$ such that $\bigcup_{D \in \mathcal{C}_{X}(k, x)} D$ is Zariski dense in $X$.

Remark 2.1. Define the function Castel $:(\mathbb{N} \backslash\{0\}) \times(\mathbb{N} \backslash\{0\}) \rightarrow \mathbb{N}$ as follows: for each $(d, n)$ with $d$ or $n$ equal to $1, \operatorname{Castel}(d, n):=0$ and, for each $(d, n)$ with $d \geq 2$ and $n \geq 2, \operatorname{Castel}(d, n):=\frac{1}{2} a(a-1)(n-1)+a b$ where $a$ and $b$ are the unique non-negative integers such that $d-1=a(n-1)+b$ and $b \leq n-2$. It is easy to see that $\operatorname{Castel}(d, n) \leq \frac{1}{2}(d-1)(d-2)$. Let $A$ be a complex algebraic curve of $\mathbb{P}^{n}(\mathbb{C})$ of degree $d$. Suppose $A$ is not contained in any hyperplane of $\mathbb{P}^{n}(\mathbb{C})$. The Castelnuovo Bound Theorem asserts that $g(A) \leq \operatorname{Castel}(d, n)$ (see page 116 of [2]). By modifying suitably the argument used in the preceding proof, we obtain the following real version of the Castelnuovo Bound Theorem: "Let $X \subset \mathbb{R}^{n}$ be a real algebraic variety of dimension $r$ and let $c:=\operatorname{cideg}\left(X, \mathbb{R}^{n}\right)$. Suppose $X$ is not contained in any affine hyperplane of $\mathbb{R}^{n}$. Then it holds: $p_{c}(X) \leq$ Castel $(c, n-r+1)$ ". This result improves Corollary 1.3.

Proof of Lemma 1.5. We will prove that each element of $\mathcal{T}(X)$ is $\leq p_{c}(X)$ completing the proof. Let $h \in \mathcal{T}(X)$. Then there exist real algebraic curves $\left\{E_{i}\right\}_{i=1}^{m}$ with $\min _{i \in\{1, \ldots, m\}} g\left(E_{i}\right)=h$ and a biregular embedding $\psi: X \rightarrow \prod_{i=1}^{m} E_{i}$. Identify $X$ with $\psi(X)$. For each $j \in\{1, \ldots, m\}$, let $\pi_{j}: \prod_{i=1}^{m} E_{i} \rightarrow E_{j}$ be the natural projection. Choose a real algebraic curve $D$ of $X$ with $g(D)=p_{c}(X)$ (which exists by definition of curve genus) and $j \in\{1, \ldots, m\}$ such that $\left.\pi_{j}\right|_{D}: D \rightarrow E_{j}$ is nonconstant. Applying Hurwitz's formula to the complexification of $\left.\pi_{j}\right|_{D}$, we infer that $g(D) \geq g\left(E_{j}\right) \geq h$. 
Remark 2.2. As an easy byproduct of the preceding proof, we obtain that, if $D$ is a real algebraic curve of a real algebraic variety $X$, then $g(D) \geq p_{t}(X)$.

Proof of Theorem 1.9. Let $X \subset \mathbb{R}^{n}, k, N, \pi$ and $\mathcal{U}$ be as in the statement of the theorem. Let $d$ be an odd integer such that $(d-1)(d-2) / 2 \geq N$ and let $\varepsilon$ be a positive real number. We denote points of $\mathbb{R}^{2 n} \times \mathbb{R}^{k}=\mathbb{R}^{n} \times \mathbb{R}^{n} \times \mathbb{R}^{k}$ by $(\tilde{x}, \tilde{y}, \tilde{t})=\left(x_{1}, \ldots, x_{n}, y_{1}, \ldots, y_{n}, t_{1}, \ldots, t_{k}\right)$. Define: the polynomial $G$ in $\mathbb{R}[x, y, \tilde{t}]$ by

$$
G(x, y, \tilde{t}):=y^{d}\left(1+\sum_{j=1}^{k} t_{j}^{2}\right)^{d}\left(1+x^{2 d}\right)-\varepsilon^{d}\left(\sum_{j=1}^{k} t_{j}^{2}\right)^{d},
$$

the algebraic subset $G^{*}$ of $\mathbb{R}^{2 n} \times \mathbb{R}^{k}$ by

$$
G^{*}:=\bigcap_{i=1}^{n}\left\{(\tilde{x}, \tilde{y}, \tilde{t}) \in \mathbb{R}^{2 n} \times \mathbb{R}^{k} \mid G\left(x_{i}, y_{i}, \tilde{t}\right)=0\right\},
$$

the Zariski closed subset $X^{*}$ of $G^{*}$ by $X^{*}:=G^{*} \cap\left(X \times \mathbb{R}^{n} \times \mathbb{R}^{k}\right)$, the regular map $\xi: X^{*} \rightarrow X$ by $\xi(\tilde{x}, \tilde{y}, \tilde{t}):=\tilde{x}$, the Nash function $f: \mathbb{R} \times \mathbb{R}^{k} \rightarrow \mathbb{R}$ by

$$
f(x, \tilde{t}):=\varepsilon\left(\sum_{j=1}^{k} t_{j}^{2}\right)\left(1+\sum_{j=1}^{k} t_{j}^{2}\right)^{-1}\left(1+x^{2 d}\right)^{-1 / d},
$$

the Nash map $\tilde{f}: \mathbb{R}^{n} \times \mathbb{R}^{k} \rightarrow \mathbb{R}^{n}$ by $\tilde{f}(\tilde{x}, \tilde{t}):=\left(f\left(x_{1}, \tilde{t}\right), \ldots, f\left(x_{n}, \tilde{t}\right)\right)$ and the Nash $k$-diffeotopy $F: \mathbb{R}^{2 n} \times \mathbb{R}^{k} \rightarrow \mathbb{R}^{2 n}$ of $\mathbb{R}^{2 n}$ by $F(\tilde{x}, \tilde{y}, \tilde{t}):=(\tilde{x}, \tilde{y}-\tilde{f}(\tilde{x}, \tilde{t}))$. For each $\tilde{t} \in \mathbb{R}^{k} \backslash\{0\}$, let $D_{\tilde{t}}$ be the nonsingular real algebraic curve of $\mathbb{R}^{2}$ defined by $D_{\tilde{t}}:=\left\{(x, y) \in \mathbb{R}^{2} \mid G(x, y, \tilde{t})=0\right\}$, let $D_{\tilde{t}}^{n} \subset \mathbb{R}^{2 n}$ be the $n^{\text {th }}$-power of $D_{\tilde{t}}$ and let $\tilde{X}_{\tilde{t}}:=X^{*} \cap \pi^{-1}(\tilde{t})$. Identify $\mathbb{R}^{n} \times \mathbb{R}^{k} \times \mathbb{R}^{n}$ with $\mathbb{R}^{2 n} \times \mathbb{R}^{k}$ by the linear isomorphism sending $(\tilde{x}, \tilde{t}, \tilde{y})$ into $(\tilde{x}, \tilde{y}, \tilde{t})$. By easy verifications, we infer that $G^{*}$ coincides with the graph of $\tilde{f}$ and the algebraic set $G^{*} \backslash \pi^{-1}(0)$ is nonsingular. It follows that the algebraic sets $X^{*} \backslash \pi^{-1}(0)$ and $\left\{\tilde{X}_{\tilde{t}}\right\}_{\tilde{t} \in \mathbb{R}^{k} \backslash\{0\}}$ are nonsingular. Moreover, since $X$ is irreducible, Lemma 1.1 of [3] implies that these algebraic sets are irreducible. The set $X^{*} \backslash \pi^{-1}(0)$ is Zariski dense in $X^{*}$ so $X^{*}$ is irreducible also. Observe that $X^{*} \cap \pi^{-1}(0)=X \times\{0\} \times\{0\}$ and, if we identify $X \subset \mathbb{R}^{n}$ with $X \times\{0\} \subset \mathbb{R}^{n} \times\{0\} \subset \mathbb{R}^{2 n}$, then $F$ extends $\xi$. Furthermore, choosing $\varepsilon$ sufficiently small, we have that $F$ is in $\mathcal{U}$. The preceding facts ensure that $\xi$ is a weak deformation of $X$ parametrized by $\left(\left.\pi\right|_{X^{*}, 0}\right)$. Fix $\tilde{t} \in \mathbb{R}^{k} \backslash\{0\}$. It remains to prove that $p_{t}\left(\widetilde{X}_{\tilde{t}}\right) \geq N$. Since $\widetilde{X}_{\tilde{t}} \subset D_{\tilde{t}}^{n}$, it suffices to show that $g\left(D_{\tilde{t}}\right) \geq N$. Let $E$ be the real Fermat curve defined as $\left\{(x, y) \in \mathbb{R}^{2} \mid x^{d}+y^{d}=1\right\}$ and let $\eta: D_{\tilde{t}} \rightarrow E$ be the regular map defined by $\eta(x, y):=\left(-x^{2}, \varepsilon y^{-1}\left(\sum_{j=1}^{k} t_{j}^{2}\right)\left(1+\sum_{j=1}^{k} t_{j}^{2}\right)^{-1}\right)$. The map $\eta$ is nonconstant so, applying Hurwitz's formula to its complexification, we infer that $g\left(D_{\tilde{t}}\right) \geq g(E)=(d-1)(d-2) / 2 \geq N$. This completes the proof. The reader observes that the preceding argument proves the singular version of Theorem 1.9 presented in Remark 1.15 as well.

Proof of Theorem 1.12. Let $k:=p_{c}(X)$ and let $x \in \operatorname{Nonsing}(X)$ such that $\bigcup_{D \in \mathcal{C}_{X}(k, x)} D$ is Zariski dense in $X$. Let us show that every regular map from $X$ 
to $Y$ is constant. Suppose that there exists a nonconstant regular map $f: X \rightarrow Y$. The Zariski closed subset $f^{-1}(f(x))$ of $X$ has dimension $<\operatorname{dim}(X)$ so there exists $D \in \mathcal{C}_{X}(k, x)$ such that $D \not \subset f^{-1}(f(x))$. It follows that the restriction of $f$ to $D$ is nonconstant. Let $E$ be the real algebraic curve of $Y$ defined as the Zariski closure of $f(D)$ in $Y$. Applying Hurwitz's formula to the complexification of the restriction of $f$ from $D$ to $E$, we obtain that $p_{c}(X)=k=g(D) \geq g(E)$. Moreover, by Remark 2.2, we know that $g(E) \geq p_{t}(Y)$. It follows that $p_{c}(X) \geq p_{t}(Y)$, which contradicts our assumption.

\subsection{Results of Subsection 1.2}

In this subsection, we assume that $X$ is an algebraic subvariety of $\mathbb{R}^{n}$ and $Y$ is an algebraic subvariety of $\mathbb{R}^{m}$.

We begin proving points (0), (1) and (2) of Theorem 1.16.

Proof of Theorem 1.16. (0) The inclusion $\operatorname{Is}\left(\mathcal{S}^{0}(X, Y)\right) \supset\left\{f \in \mathcal{S}^{0}(X, Y) \mid f(X) \subset\right.$ Is $(Y)\}$ is evident. Since $Y$ is an absolute neighborhood retract, it is easy to prove that, if $f$ is an element of $\mathcal{S}^{0}(X, Y)$ with $f(X) \not \subset \operatorname{Is}(Y)$, then $f$ is not isolated in $\mathcal{S}^{0}(X, Y)$ (see the proof of point (2) below). It follows that $\operatorname{Is}\left(\mathcal{S}^{0}(X, Y)\right)$ is equal to $\left\{f \in \mathcal{S}^{0}(X, Y) \mid f(X) \subset \operatorname{Is}(Y)\right\}$. In particular, $\operatorname{Is}\left(\mathcal{S}^{0}(X, Y)\right)$ is finite because $X$ and $Y$ have finitely many semi-algebraically connected components. The remainder of point ( 0 ) is evident.

(1) By Theorem 1.12, $\mathcal{R}(X, Y)$ coincides with the set of all constant maps from $X$ to $Y$. This fact implies that $\mathcal{R}(X, Y)$ is closed in $\mathcal{S}^{0}(X, Y)$ and is homeomorphic to $Y$. In order to prove that $\mathcal{R}(X, Y)$ is nowhere dense up to isolated points in $\mathcal{S}^{0}(X, Y)$, one can follow the argument used in the proof of point (2) below.

(2) The proof of this point is based on an idea of Bochnak and Kucharz (see [5, page 1572]). By hypothesis, the toric genus of $Y$ is $\geq 1$ so we may suppose that $Y$ is an algebraic subvariety of some product variety $\prod_{i=1}^{a} E_{i} \subset \mathbb{R}^{m}$ where $\left\{E_{i}\right\}_{i=1}^{a}$ are real algebraic curves with $\min _{i \in\{1, \ldots, a\}} g\left(E_{i}\right) \geq 1$. For each $j \in\{1, \ldots, a\}$, let $\pi_{j}: \prod_{i=1}^{a} E_{i} \rightarrow E_{j}$ be the natural projection. We must prove that, for each $f \in \mathcal{R}(X, Y) \backslash \operatorname{Is}\left(\mathcal{S}^{0}(X, Y)\right)$ and for each neighborhood $\mathcal{U}$ of $f$ in $\mathcal{S}^{0}(X, Y)$, there exists $g \in \mathcal{U}$, which is not approximable by regular maps in $\mathcal{S}^{0}(X, Y)$. Let $f$ and $\mathcal{U}$ be as above. Let $\Omega$ be an open semi-algebraic neighborhood of $Y$ in $\mathbb{R}^{m}$ and let $\rho: \Omega \rightarrow Y$ be a continuous semi-algebraic retraction. Fix $x \in \operatorname{Nonsing}(X)$. Since $f \notin \operatorname{Is}\left(\mathcal{S}^{0}(X, Y)\right)$, we have that $f(x)$ is not an isolated point of $Y$. Define $y:=$ $f(x)$ and choose a nonsingular real algebraic curve $D$ of $X$ containing $x$. By the Nash curve selection lemma (Proposition 8.1.13 of [4]), we can find a real algebraic curve $E$ of $Y$ such that $y$ is contained in the (strong) closure of $\operatorname{Nonsing}(E)$ in $Y$. Let $U$ be a small open semi-algebraic neighborhood of $x$ in $\operatorname{Nonsing}(X)$ and let $V$ be a small open semi-algebraic neighborhood of $y$ in $\mathbb{R}^{m}$ such that $f(U) \subset V \cap Y$, $U \cap D$ is Nash isomorphic to $\mathbb{R}$ and $V \cap \operatorname{Nonsing}(E)$ contains an open semi-algebraic subset $V^{\prime}$ of Nonsing $(E)$ Nash isomorphic to $\mathbb{R}$. Restricting $V^{\prime}$ if needed, we may also suppose that the restriction of some projection $\pi_{j}$ to $V^{\prime}$ is a Nash isomorphism 
into an open semi-algebraic subset of Nonsing $\left(E_{j}\right)$. By using an adequate semialgebraic partition of unity on $X$, it is now easy to construct a continuous semialgebraic map $g^{*}: X \rightarrow \Omega$ such that: $g^{*}=f$ on $X \backslash U, g^{*}(U) \subset V$ and there exists a non-empty open semi-algebraic subset $U^{\prime}$ of $U \cap D$ such that $g^{*}\left(U^{\prime}\right) \subset V^{\prime}$ and the restriction of $g^{*}$ from $U^{\prime}$ to $V^{\prime}$ is a nonconstant Nash map with at least $\ell:=\max \{0,2 g(D)-1\}$ critical points, each of multiplicity exactly 2 . Define the continuous semi-algebraic map $g: X \rightarrow Y$ by $g:=\rho \circ g^{*}$. Observe that the restriction of the composition map $\left(\left.\pi_{j}\right|_{Y}\right) \circ g: X \rightarrow E_{j}$ to $U^{\prime}$ is a nonconstant Nash map with at least $\ell$ critical points, each of multiplicity exactly 2 . Choosing $V$ (and hence $U$ ) sufficiently small, we have that $g \in \mathcal{U}$. It remains to show that $g$ is not approximable by regular maps in $\mathcal{S}^{0}(X, Y)$. Suppose this is false. Then there exists $\bar{g} \in \mathcal{R}(X, Y)$ such that the restriction $\eta: D \rightarrow E_{j}$ of the composition map $\left(\left.\pi_{j}\right|_{Y}\right) \circ \bar{g}: X \rightarrow E_{j}$ to $D$ is a nonconstant regular map with at least $\ell$ critical points. Applying Hurwitz's formula to the complexification $\eta_{\mathbb{C}}$ of $\eta$, we infer that $g(D) \geq g\left(E_{j}\right) \geq 1, \ell=2 g(D)-1$ and the degree $v\left(\eta_{\mathbb{C}}\right)$ of the ramification divisor of $\eta_{\mathbb{C}}$ is $\leq 2 g(D)-2=\ell-1$. This is impossible because, by construction, $v\left(\eta_{\mathbb{C}}\right) \geq \ell$.

In order to prove point (3) of Theorem 1.16, we need some preliminary results.

Lemma 2.3. Let $X$ be a real algebraic variety and let $E$ be a real algebraic curve with $g(E) \geq 2$. Then $\operatorname{Dom}(X, E)$ is finite and it holds:

$$
\sharp \operatorname{Dom}(X, E) \leq \mathcal{M}\left(p_{c}(X), g(E)\right) .
$$

Proof. Let $h:=\mathcal{M}\left(p_{c}(X), g(E)\right)$. Suppose $\sharp \operatorname{Dom}(X, E)>h$ and let $f_{0}, \ldots, f_{h}$ be distinct elements of $\operatorname{Dom}(X, E)$. Let $k:=p_{c}(X)$ and let $x \in \operatorname{Nonsing}(X)$ such that $\bigcup_{D \in \mathcal{C}_{X}(k, x)} D$ is Zariski dense in $X$. Define

$$
\Delta:=\bigcup_{i=0}^{h} f_{i}^{-1}\left(f_{i}(x)\right) \cup \bigcup_{i \neq j}\left\{p \in X \mid f_{i}(p)=f_{j}(p)\right\} .
$$

Since $\Delta$ is a proper Zariski closed subset of $X$, there exists $D \in \mathcal{C}_{X}(k, x)$ such that $D \not \subset \Delta$. It follows that the maps $\left.f_{0}\right|_{D}, \ldots,\left.f_{h}\right|_{D}$ are $h+1$ distinct elements of $\operatorname{Dom}(D, E)$. In particular, their complexifications are $h+1$ distinct elements of $\operatorname{Dom}_{\mathbb{C}}\left(D_{\mathbb{C}}, E_{\mathbb{C}}\right)$. This contradicts the de Franchis-Martens theorem.

We fix a convention: the dimension of the empty set is equal to -1 .

Lemma 2.4. Let $V$ and $W$ be algebraic subsets of $\mathbb{R}^{n}$ and of $\mathbb{R}^{m}$ respectively and let $f: V \rightarrow W$ be a regular map. For each $k \in \mathbb{N}$, the set $T_{k}(f):=\{w \in$ $\left.W \mid \operatorname{dim}\left(f^{-1}(w)\right) \geq k\right\}$ is semi-algebraic.

Proof. Fix $k \in \mathbb{N}$. Let us proceed by induction on $v:=\operatorname{dim}(V)$. The case $v=0$ is evident. Let $v \geq 1$. Indicate by $V_{1}, \ldots, V_{q}$ the irreducible components of $V$. Since $T_{k}(f)=\bigcup_{i=1}^{q} T_{k}\left(\left.f\right|_{V_{i}}\right)$, we may suppose that $V$ is irreducible. Replacing $W$ with the Zariski closure of $f(V)$ in $W$, we may suppose that $f$ is dominating also. By Sard's theorem, there exists a Zariski closed subset $Z$ of $W$ such that $W \backslash Z \subset$ 
$\operatorname{Nonsing}(W), \operatorname{dim}(Z)<\operatorname{dim}(W)$ and the restriction $g$ of $f$ from $\operatorname{Nonsing}(V) \backslash$ $f^{-1}(Z)$ to $W \backslash Z$ is a submersion. It follows that $T_{k}(g)$ is semi-algebraic. In fact, $T_{k}(g)=f(\operatorname{Nonsing}(V)) \backslash Z$ if $k \leq v-\operatorname{dim}(W)$ and $T_{k}(g)=\emptyset$ if $k>v-\operatorname{dim}(W)$. Let $V^{*}:=(V \backslash \operatorname{Nonsing}(V)) \cup f^{-1}(Z)$. Since $V^{*}$ is an algebraic subset of $\mathbb{R}^{n}$ of dimension $<v$ and $T_{k}(f)=T_{k}(g) \cup T_{k}\left(\left.f\right|_{V^{*}}\right)$, by induction, it follows that $T_{k}(f)$ is semi-algebraic.

Proof of Theorem 1.16, (3). By hypothesis, the toric genus of $Y$ is $\geq 2$ so there exist real algebraic curves $\left\{E_{i}\right\}_{i=1}^{a}$ with $\min _{i \in\{1, \ldots, a\}} g\left(E_{i}\right) \geq 2$ and a biregular embedding $\psi$ of $Y$ into the product variety $T:=\prod_{i=1}^{a} E_{i}$. Identify $Y$ with $\psi(Y)$. By Lemma 2.3, we know that $\operatorname{Dom}\left(X, E_{i}\right)$ is finite for each $i \in\{1, \ldots, a\}$. Let $L$ be the set of all $i \in\{1, \ldots, a\}$ such that $\operatorname{Dom}\left(X, E_{i}\right)$ is non-empty. If $L$ is empty, then every map from $X$ to $T$ (and hence to $Y$ ) is constant. In this case, point (3) follows from point (1). Let $L$ be non-empty. We may suppose that $L=\{1, \ldots, b\}$ for some $b \in\{1, \ldots, a\}$. For each $i \in\{1, \ldots, a\}$, let $\pi_{i}: T \rightarrow E_{i}$ be the natural projection and, for each $i \in\{1, \ldots, b\}$, let $f_{i, 1}, \ldots, f_{i, n_{i}}$ be the elements of $\operatorname{Dom}\left(X, E_{i}\right)$. Indicate by $\mathcal{C}$ the set of all constant maps from $X$ to $Y$. For each non-empty subset $\chi$ of $\{1, \ldots, b\}$, let $F(\chi)$ be the set of all functions $\eta: \chi \rightarrow \mathbb{N}$ such that $\eta(i) \in$ $\left\{1, \ldots, n_{i}\right\}$ for each $i \in \chi$, let $T_{\chi}:=\prod_{i \in \chi} E_{i}$, let $\chi^{\prime}:=\{1, \ldots, a\} \backslash \chi$ and let $\pi_{\chi^{\prime}}: T \rightarrow T_{\chi^{\prime}}$ be the natural projection (where $T_{\emptyset}$ is considered equal to a point). For each non-empty subset $\chi$ of $\{1, \ldots, b\}$ and for each $\eta \in F(\chi)$, define

$$
\mathcal{S}_{\chi, \eta}:=\left\{f \in \mathcal{R}(X, T) \mid \pi_{i} \circ f=f_{i, \eta(i)} \text { for each } i \in \chi, \pi_{\chi^{\prime}} \circ f \text { is constant }\right\}
$$

and $\mathcal{R}_{\chi, \eta}$ as the set of all maps $f \in \mathcal{S}_{\chi, \eta}$ with $f(X) \subset Y$, viewed as maps from $X$ to $Y$. It is immediate to see that $\mathcal{C}$ and all sets of the form $\mathcal{R}_{\chi, \eta}$ cover $\mathcal{R}(X, Y)$, are pairwise disjoint and are open in $\mathcal{R}(X, Y)$. In other words, they form an open partition of $\mathcal{R}(X, Y)$. Observe that $\mathcal{C}$ is closed in $\mathcal{S}^{0}(X, Y)$ and is homeomorphic to $Y$. In this way, it suffices to prove that each $\mathcal{R}_{\chi, \eta}$ is closed in $\mathcal{S}^{0}(X, Y)$ and is homeomorphic to a closed semi-algebraic set, which turns out to be bounded if $Y$ is bounded. Fix a non-empty subset $\chi$ of $\{1, \ldots, b\}$ and $\eta \in F(\chi)$. The set $\mathcal{R}_{\chi, \eta}$ is closed in $\mathcal{S}^{0}(X, Y)$ because it is evident that its complement in $\mathcal{S}^{0}(X, Y)$ is open. Define $h: \mathcal{R}_{\chi, \eta} \rightarrow T_{\chi^{\prime}}$ by $h(f):=\pi_{\chi^{\prime}}(f(x)$ ) for some (and hence for every) point $x \in X$. By a standard verification, we infer that $h$ is a homeomorphism into its image. We will prove that $h\left(\mathcal{R}_{\chi, \eta}\right)$ is a closed semi-algebraic subset of $T_{\chi^{\prime}}$. Identify $T_{\chi} \times T_{\chi^{\prime}}$ with $T$. Let $F: X \rightarrow T_{\chi}$ be the regular map such that $\pi_{i} \circ F=f_{i, \eta(i)}$ for each $i \in \chi$, let $V$ be the Zariski closure of $F(X)$ in $T_{\chi}$ and let $v:=\operatorname{dim}(V)$. Observe that $p \in h\left(\mathcal{R}_{\chi, \eta}\right)$ if and only if $V \times\{p\} \subset Y \cap \pi_{\chi^{\prime}}^{-1}(p)$ or, equivalently, $\operatorname{dim}\left((V \times\{p\}) \cap\left(Y \cap \pi_{\chi^{\prime}}^{-1}(p)\right)\right) \geq v$. Let $V^{*}:=\left(V \times T_{\chi^{\prime}}\right) \cap Y$ and let $\xi: V^{*} \rightarrow T_{\chi^{\prime}}$ be the restriction of $\pi_{\chi^{\prime}}$ to $V^{*}$. For each $p \in T_{\chi^{\prime}}$, the fiber of $\xi$ over $p$ is equal to $(V \times\{p\}) \cap\left(Y \cap \pi_{\chi^{\prime}}^{-1}(p)\right)$ so, applying Lemma 2.4 to $\xi$, we obtain that $h\left(\mathcal{R}_{\chi, \eta}\right)=T_{\nu}(\xi)$ is semi-algebraic. Let us prove that $h\left(\mathcal{R}_{\chi, \eta}\right)$ is closed in $T_{\chi^{\prime}}$. For each $q \in T_{\chi^{\prime}}$, let $f_{q}$ be the map in $\mathcal{S}_{\chi, \eta}$ defined by $f_{q}(x):=(F(x), q)$. Fix $q^{\prime} \in T_{\chi^{\prime}} \backslash h\left(\mathcal{R}_{\chi, \eta}\right)$. Then there exists $x \in X$ such that $f_{q^{\prime}}(x) \notin Y$. Since $Y$ 
is closed in $T$ (algebraic subvarieties are assumed to be Zariski closed, and hence strong closed), there exists a neighborhood $U$ of $q^{\prime}$ in $T_{\chi^{\prime}}$ such that $f_{q}(x) \notin Y$ for each $q \in U$. It follows that $U \cap h\left(\mathcal{R}_{\chi, \eta}\right)$ is empty. Finally, suppose $Y$ bounded. Since $h\left(\mathcal{R}_{\chi, \eta}\right) \subset \pi_{\chi^{\prime}}(Y)$, we have that $h\left(\mathcal{R}_{\chi, \eta}\right)$ is bounded also. The proof is complete.

Suppose now $X$ and $Y$ nonsingular.

Proof of Theorem 1.17. The proofs of points (1) and (3) of Theorem 1.16 continue to work if we replace $\mathcal{S}^{0}(X, Y)$ with $\mathcal{N}(X, Y)$ equipped with the $C^{\infty}$ compact-open topology. In this way, points (1) and (3) of Theorem 1.17 hold, except for the fact that $\mathcal{R}(X, Y)$ is nowhere dense in $\mathcal{N}(X, Y)$ when $p_{t}(Y) \geq 2$. The proof of the latter fact can be easily obtained by the argument used in the proof of point (2) below. Let us prove such a point following the proof of point (2) of Theorem 1.16. Since $p_{t}(Y) \geq 1$, we may suppose that $Y$ is an algebraic subvariety of $\prod_{i=1}^{a} E_{i} \subset \mathbb{R}^{m}$ where $\left\{E_{i}\right\}_{i=1}^{a}$ are real algebraic curves with $\min _{i \in\{1, \ldots, a\}} g\left(E_{i}\right) \geq 1$. For each $j \in$ $\{1, \ldots, a\}$, let $\pi_{j}: \prod_{i=1}^{a} E_{i} \rightarrow E_{j}$ be the natural projection. We must prove that, for each $f \in \mathcal{N}(X, Y)$ such that $\operatorname{dim}\left(f^{-1}(y)\right) \geq 1$ for some $y \in Y$ and for each neighborhood $\mathcal{U}$ of $f$ in $\mathcal{N}(X, Y)$, there exists $g \in \mathcal{U}$, which is not approximable by regular maps in $\mathcal{N}(X, Y)$. Define $r:=\operatorname{dim}(X)$ and $s:=\operatorname{dim}(Y)$. Let $f, y$ and $\mathcal{U}$ be as above, let $E$ be a nonsingular algebraic curve of $Y$ containing $y$ and let $D$ be a real algebraic curve of $X$ such that $f^{-1}(y) \cap \operatorname{Nonsing}(D)$ contains a non-empty open semi-algebraic subset $\Omega$ of Nonsing $(D)$. Fix $x \in \Omega$. Choose an open semialgebraic neighborhood $U$ of $x$ in $X$ and an open semi-algebraic neighborhood $V$ of $y$ in $Y$ such that $U \cap D \subset \Omega, f(U) \subset V$ and there exist Nash isomorphisms $\alpha: U \rightarrow \mathbb{R}^{r}$ and $\beta: V \rightarrow \mathbb{R}^{s}$ with the following properties: $\alpha(U \cap D)=$ $\mathbb{R} \times\{0\} \subset \mathbb{R} \times \mathbb{R}^{r-1}=\mathbb{R}^{r}, \beta(y)=0$ and $\beta(V \cap E)=\mathbb{R} \times\{0\} \subset \mathbb{R} \times \mathbb{R}^{s-1}=\mathbb{R}^{s}$. We may also suppose that the restriction of some projection $\pi_{j}$ to $\beta^{-1}((0,3) \times\{0\})$ is a Nash isomorphism into an open semi-algebraic subset of $\operatorname{Nonsing}\left(E_{j}\right)$. Let $k$ be a positive integer and let $\varepsilon$ be a real number contained in $(0,1)$. Choose a semialgebraic function $g_{k}: \mathbb{R}^{r} \rightarrow \mathbb{R}$ of class $C^{k}$ such that $g_{k}$ vanishes on $\mathbb{R}^{r} \backslash(-2,2)^{r}$, $g_{k}((-1,1) \times\{0\}) \subset(1,2)$ and the restriction of $g_{k}$ to $(-1,1) \times\{0\}$ is nonconstant and has at least $\ell:=\max \{0,2 g(D)-1\}$ critical points, each of multiplicity exactly 2. Define the semi-algebraic maps $f^{*}, g_{k}^{*}$ and $f_{k, \varepsilon}^{*}$ from $\mathbb{R}^{r}$ to $\mathbb{R}^{s}$ of class $C^{k}$ as follows: $f^{*}:=\left.\beta \circ f\right|_{U} \circ \alpha^{-1}, g_{k}^{*}(p):=\left(g_{k}(p), 0, \ldots, 0\right)$ for each $p \in \mathbb{R}^{r}$ and $f_{k, \varepsilon}^{*}:=f^{*}+\varepsilon g_{k}^{*}$. Observe that $f_{k, \varepsilon}^{*}=\varepsilon g_{k}^{*}$ on $(-1,1) \times\{0\}$ and $f_{k, \varepsilon}^{*}((-1,1) \times\{0\}) \subset$ $(0,3) \times\{0\}$. Let $f_{k, \varepsilon}: X \rightarrow Y$ be the semi-algebraic map of class $C^{k}$ defined by setting $f_{k, \varepsilon}:=f$ on $X \backslash U$ and $f_{k, \varepsilon}:=\beta^{-1} \circ f_{k, \varepsilon}^{*} \circ \alpha$ on $U$. By [27, Theorem II.4.1], we can find a map $g$ in $\mathcal{N}(X, Y)$ arbitrarily close to $f_{k, \varepsilon}$ with respect to the $C^{k}$ compact-open topology (see [14, 27] and [10, page 351]). Choosing $k$ sufficiently large, $\varepsilon$ sufficiently small and $g$ sufficiently close to $f_{k, \varepsilon}$, we have that $g \in \mathcal{U}$ and the restriction of the composition map $\left(\left.\pi_{j}\right|_{Y}\right) \circ g: X \rightarrow E_{j}$ to $\alpha^{-1}((-1,1) \times\{0\})$ has image contained in $\operatorname{Nonsing}\left(E_{j}\right)$, is nonconstant and has at least $\ell$ critical points. In order to prove that $g$ is not approximable by regular maps in $\mathcal{N}(X, Y)$, it suffices to repeat word for word the last part of the proof of point (2) of Theorem 1.16. 


\subsection{Results of Subsection 1.3}

We need some preparations.

Definition 2.5. Let $X$ be a real algebraic variety. The toric $\operatorname{dimension} \operatorname{tdim}(X)$ of $X$ is the minimum integer $n \in \mathbb{N}$ such that there exist real algebraic curves $D_{1}, \ldots, D_{n}$ with $\min _{i \in\{1, \ldots, n\}} g\left(D_{i}\right)=p_{t}(X)$ and a biregular embedding of $X$ into $\prod_{i=1}^{n} D_{i}$.

The toric dimension is a biregular invariant on real algebraic varieties. If $X$ is a real algebraic curve, then $\operatorname{tdim}(X)=1$. More precisely, if $X$ is the product variety of $n$ real algebraic curves, then $\operatorname{tdim}(X)=n$ (see Example 1.6,(ii)).

Lemma 2.6. Let $X$ and $Y$ be real algebraic varieties. If $p_{t}(Y) \geq 2$, then $\operatorname{Dom}(X, Y)$ is finite and it holds:

$$
\sharp \operatorname{Dom}(X, Y) \leq \mathcal{M}_{*}\left(p_{c}(X), p_{t}(Y)\right)^{\operatorname{tdim}(Y)} .
$$

Proof. Define $n:=\operatorname{tdim}(Y)$. Let $\left\{D_{i}\right\}_{i=1}^{n}$ be real algebraic curves such that $\min _{i \in\{1, \ldots, n\}} g\left(D_{i}\right)=p_{t}(Y) \geq 2$ and there exists a biregular embedding $\psi$ of $Y$ into $\prod_{i=1}^{n} D_{i}$. Identify $Y$ with $\psi(Y)$ and indicate by $e: Y \hookrightarrow \prod_{i=1}^{n} D_{i}$ the inclusion map. For each $j \in\{1, \ldots, n\}$, let $\pi_{j}: \prod_{i=1}^{n} D_{i} \rightarrow D_{j}$ be the natural projection. Identify $\mathcal{R}\left(X, \prod_{i=1}^{n} D_{i}\right)$ with $\prod_{i=1}^{n} \mathcal{R}\left(X, D_{i}\right)$ in the natural way and define the injective map $\xi: \operatorname{Dom}(X, Y) \rightarrow \prod_{i=1}^{n} \mathcal{R}\left(X, D_{i}\right)$ by $\xi(f):=e \circ f$. By Lemma 2.3, we know that, for each $i \in\{1, \ldots, n\}$, $\operatorname{Dom}\left(X, D_{i}\right)$ is finite and $\sharp \operatorname{Dom}\left(X, D_{i}\right) \leq \mathcal{M}\left(p_{c}(X), g\left(D_{i}\right)\right) \leq \mathcal{M}_{*}\left(p_{c}(X), p_{t}(Y)\right)$. In this way, it suffices to prove that the image of $\xi$ is contained in $\prod_{i=1}^{n} \operatorname{Dom}\left(X, D_{i}\right)$. On the contrary, suppose this is false. Let $f \in \operatorname{Dom}(X, Y)$ such that $\xi(f) \notin \prod_{i=1}^{n} \operatorname{Dom}\left(X, D_{i}\right)$. Then there exist $j \in\{1, \ldots, n\}$ and $q \in D_{j}$ such that $\pi_{j} \circ e \circ f$ is constantly equal to $q$ or, equivalently, $f(X) \subset \pi_{j}^{-1}(q)$. Since $f$ is dominating, it follows that $Y \subset \pi_{j}^{-1}(q)$. Up to rearrange the indices, we may suppose that $j=n$. Identify $\pi_{j}^{-1}(q)$ with $\prod_{i=1}^{n-1} D_{i}$. By the definition of toric genus, we have that $p_{t}(Y) \geq \min _{i \in\{1, \ldots, n-1\}} g\left(D_{i}\right)$ so it holds: $p_{t}(Y)=\min _{i \in\{1, \ldots, n-1\}} g\left(D_{i}\right)$. This fact and the inclusion $Y \subset \prod_{i=1}^{n-1} D_{i}$ contradicts the definition of $n$.

In the next two lemmas, we show that, if a real algebraic variety $X$ has toric genus $\geq 2$, then $\operatorname{tdim}(X) \leq \mathcal{F}\left(p_{c}(X)\right)$. This result and Lemma 2.6 prove Theorem 1.18 .

Let $X$ be a real algebraic variety and let $G(X)$ be the set of dominating regular maps from $X$ to some real algebraic curve $D$ with $g(D) \geq 2$. Define an equivalence relation $\mathcal{H}$ on $G(X)$ as follows: $f \in \operatorname{Dom}(X, D)$ is $\mathcal{H}$-equivalent to $f^{\prime} \in \operatorname{Dom}\left(X, D^{\prime}\right)$ in $G(X)$ if and only if there is a biregular isomorphism $h: D \rightarrow D^{\prime}$ such that $f^{\prime}=h \circ f$. Indicate by $\mathcal{G}(X)$ the set of all $\mathcal{H}$-equivalence classes of $G(X)$.

Lemma 2.7. Let $X$ be a real algebraic variety with $p_{t}(X) \geq 2$. Then $\mathcal{G}(X)$ is finite and it holds: $\sharp \mathcal{G}(X) \leq \mathcal{F}\left(p_{c}(X)\right)$. 
Proof. We will follow the proof of Lemma 2.3. Let $h:=\mathcal{F}\left(p_{c}(X)\right)$. Suppose $\sharp \mathcal{G}(X)>h$ and let $f_{0}: X \rightarrow E_{0}, \ldots, f_{h}: X \rightarrow E_{h}$ be elements of $G(X)$ representing distinct $\mathcal{H}$-equivalence classes. Let $k:=p_{c}(X)$ and let $x \in \operatorname{Nonsing}(X)$ such that $\bigcup_{D \in \mathcal{C}_{X}(k, x)} D$ is Zariski dense in $X$. Let $A$ be the set of all pairs $(i, j)$ of integers in $\{0,1, \ldots, h\}$ such that $i<j$ and $E_{i}$ is biregularly isomorphic to $E_{j}$. For each $(i, j) \in A$, let $B_{i j}$ be the set of all biregular isomorphisms from $E_{i}$ to $E_{j}$. By Lemma 2.3, we know that each $B_{i j}$ is finite. For each $(i, j) \in A$, let $\psi_{i j 1}, \ldots, \psi_{i, j, n_{i j}}$ be the elements of $B_{i j}$. Define

$$
\Delta:=\bigcup_{i=0}^{h} f_{i}^{-1}\left(f_{i}(x)\right) \cup \bigcup_{(i, j) \in A} \bigcup_{\ell=1}^{n_{i j}}\left\{p \in X \mid\left(\psi_{i j \ell} \circ f_{i}\right)(p)=f_{j}(p)\right\} .
$$

Since $\Delta$ is a proper Zariski closed subset of $X$, there exists $D \in \mathcal{C}_{X}(k, x)$ such that $D \not \subset \Delta$. It follows that the maps $\left.f_{0}\right|_{D}, \ldots,\left.f_{h}\right|_{D}$ represent $h+1$ distinct $\mathcal{H}$ equivalence classes of $G(D)$. In particular, their complexifications represent $h+1$ distinct $\mathcal{H}_{\mathbb{C}}$-equivalence classes of $G_{\mathbb{C}}\left(D_{\mathbb{C}}\right)$. This contradicts the improved version of the de Franchis-Severi theorem due to Howard and Sommese [15], which we have recalled in Subsection 1.3.

Lemma 2.8. Let $X$ be as above. Then it holds: $\operatorname{tdim}(X) \leq \mathcal{F}\left(p_{c}(X)\right)$.

Proof. Let $n:=\operatorname{tdim}(X)$ and let $m:=\sharp \mathcal{G}(X)$. By Lemma 2.7, it suffices to show that $n \leq m$. On the contrary, suppose $m<n$. Let $\left\{D_{i}\right\}_{i=1}^{n}$ be real algebraic curves such that $\min _{i \in\{1, \ldots, n\}} g\left(D_{i}\right)=p_{t}(X) \geq 2$ and there exists a biregular embedding $\psi$ of $X$ into $\prod_{i=1}^{n} D_{i}$. Identify $X$ with $\psi(X)$. For each $j \in\{1, \ldots, n\}$, let $f_{j}: X \rightarrow D_{j}$ be the restriction to $X$ of the natural projection of $\prod_{i=1}^{n} D_{i}$ onto $D_{j}$. Let $\rho: G(X) \rightarrow \mathcal{G}(X)$ be the natural projection and let $\alpha_{1}, \ldots, \alpha_{k}$ be the elements of the set $\rho\left(\left\{f_{1}, \ldots, f_{n}\right\}\right)$. Up to rearrange the indices, we may suppose that there exist integers $\left\{n_{i}\right\}_{i=1}^{k+1}$ such that $1=n_{1}<n_{2}<\cdots<n_{k}<n_{k+1}=n+1$ and, for each $i \in\{1, \ldots, k\}, \rho^{-1}\left(\alpha_{i}\right)=\left\{f_{n_{i}}, f_{n_{i}+1}, \ldots, f_{n_{i+1}-1}\right\}$. For each $i \in$ $\{1, \ldots, k\}$ and for each $j \in\left\{n_{i}, n_{i}+1, \ldots, n_{i+1}-1\right\}$, let $\psi_{i j}: D_{j} \rightarrow D_{n_{i}}$ be a biregular isomorphism such that $f_{n_{i}}=\psi_{i j} \circ f_{j}$ (where $\psi_{i, n_{i}}$ is considered equal to the identity map on $D_{n_{i}}$ ). For each $i \in\{1, \ldots, k\}$, indicate by $s_{i}$ the positive integer $n_{i+1}-n_{i}$ and by $D_{n_{i}}^{s_{i}}$ the $s_{i}^{\text {th }}$-power of $D_{n_{i}}$, and define the real algebraic curve $\Delta_{i}$ by $\Delta_{i}:=\left\{\left(x_{1}, x_{2}, \ldots, x_{s_{i}}\right) \in D_{n_{i}}^{s_{i}} \mid x_{1}=x_{2}=\ldots=x_{s_{i}}\right\}$. Identify $\prod_{i=1}^{n} D_{i}$ with $\prod_{i=1}^{k} \prod_{j=n_{i}}^{n_{i+1}-1} D_{j}$. Let $\Psi: \prod_{i=1}^{k} \prod_{j=n_{i}}^{n_{i+1}-1} D_{j} \rightarrow \prod_{i=1}^{k} D_{n_{i}}^{s_{i}}$ be the biregular isomorphism defined by $\Psi:=\prod_{i=1}^{k} \prod_{j=n_{i}}^{n_{i+1}-1} \psi_{i j}$. Identify $X$ with $\Psi(X)$. Since $f_{n_{i}}=\psi_{i j} \circ f_{j}$ for each $i \in\{1, \ldots, k\}$ and for each $j \in\left\{n_{i}, n_{i}+1, \ldots, n_{i+1}-1\right\}$, it follows that $X$ is contained in the subset $\prod_{i=1}^{k} \Delta_{i}$ of $\prod_{i=1}^{k} D_{n_{i}}^{s_{i}}$. By the definition of toric genus, it follows that $p_{t}(X) \geq \min _{i \in\{1, \ldots, k\}} g\left(\Delta_{i}\right)$. On the other hand, $\min _{i \in\{1, \ldots, k\}} g\left(\Delta_{i}\right) \geq \min _{i \in\{1, \ldots, n\}} g\left(D_{i}\right)=p_{t}(X)$. Summarizing, we have that $X \subset \prod_{i=1}^{k} \Delta_{i}$ and $\min _{i \in\{1, \ldots, k\}} g\left(\Delta_{i}\right)=p_{t}(X)$. Since $k \leq m<n$, the latter facts contradict the definition of $n$.

Remark 2.9. Let $X$ be a $r$-dimensional real algebraic variety with $p_{t}(X) \geq 2$. Since $r \leq \operatorname{tdim}(X)$, it follows that $r \leq \mathcal{F}\left(p_{c}(X)\right)$. Recall that $\mathcal{F}(2)=1$. In this way, if $p_{c}(X)=2$ (and hence $p_{t}(X)=2$ also), then $r=1$. 
Before giving the proof of Theorem 1.19, we fix some notations. Let $X$ be a real algebraic variety, let $S$ be a subset of $X$ and let $Z$ be a Zariski closed subset of $X$. We denote by $\operatorname{Zcl}_{X}(S)$ the Zariski closure of $S$ in $X$ and by $\operatorname{Cent}(Z)$ the (strong) closure in $Z$ of the set of nonsingular points of $Z$ of maximum dimension.

Proof of Theorem 1.19. Define two families $\left\{Y_{i}\right\}_{i=1}^{k}$ and $\left\{Y_{i}^{\prime}\right\}_{i=1}^{k}$ of subsets of $Y$ as follows:

$$
\left\{\begin{array}{l}
Y_{1}:=Y, \quad Y_{1}^{\prime}:=\operatorname{Cent}(Y), \\
Y_{i+1}:=\operatorname{Zcl}_{Y}\left(Y_{i} \backslash \bigcup_{j=1}^{i} Y_{j}^{\prime}\right), \quad Y_{i+1}^{\prime}:=\operatorname{Cent}\left(Y_{i+1}\right) \backslash \bigcup_{j=1}^{i} Y_{j}^{\prime}, \\
Y_{k} \neq \emptyset, \quad Y_{k+1}=\emptyset .
\end{array}\right.
$$

Observe that, for each $i \in\{1, \ldots, k\}, \operatorname{dim}\left(Y_{i+1}\right)<\operatorname{dim}\left(Y_{i}\right)$ so the preceding definition is consistent. It is easy to verify that $\left\{Y_{i}^{\prime}\right\}_{i=1}^{k}$ is a partition of $Y$ and, for each $i \in$ $\{1, \ldots, k\}, Y_{i}^{\prime}$ is non-empty, $\bigcup_{j=1}^{i} Y_{j}^{\prime}$ is closed in $Y$ and $Y_{i} \backslash \bigcup_{j=1}^{i-1} Y_{j}^{\prime}=Y \backslash \bigcup_{j=1}^{i-1} Y_{j}^{\prime}$ where $\bigcup_{j=1}^{0} Y_{j}^{\prime}$ is considered equal to the empty set. For each $i \in\{1, \ldots, k\}$, let $Y_{i 1}, \ldots, Y_{i, n_{i}}$ be the irreducible components of $Y_{i}$ of maximum dimension. Let $f \in \operatorname{wDom}(X, Y)$ and let $U$ be the interior of the closure of $f(\operatorname{Nonsing}(X))$ in $Y$. Define $h$ as the minimum integer $i \in\{1, \ldots, k\}$ such that $U \cap Y_{i}^{\prime} \neq \varnothing$ and let $V:=U \cap\left(Y_{h} \backslash \bigcup_{j=1}^{h-1} Y_{j}^{\prime}\right)$. Since $\emptyset \neq U \cap Y_{h}^{\prime} \subset V=U \cap\left(Y \backslash \bigcup_{j=1}^{h-1} Y_{j}^{\prime}\right)$, we have that $V$ is a non-empty open semi-algebraic subset of $Y$ contained in $Y_{h}$ and $f^{-1}(V) \cap \operatorname{Nonsing}(X)$ is a non-empty open semi-algebraic subset of $\operatorname{Nonsing}(X)$. In particular, we infer that $f^{-1}\left(Y_{h}\right)=X$ or, equivalently, $f(X) \subset Y_{h}$. Let $A$ be the Zariski closure of $f(X)$ in $Y_{h}$. Since the interior of $A$ in $Y_{h}$ contains $V$ and $V$ intersects $\operatorname{Cent}\left(Y_{h}\right)$, it follows that $A=Y_{h j}$ for some (unique) $j \in\left\{1, \ldots, n_{h}\right\}$. Let $f_{*}: X \rightarrow Y_{h j}$ be the restriction of $f$ from $X$ to $Y_{h j}$. Define the injective map $\xi: \operatorname{wDom}(X, Y) \rightarrow \bigsqcup_{i=1}^{k} \bigsqcup_{j=1}^{n_{i}} \operatorname{Dom}\left(X, Y_{i j}\right)$ (the symbol “ $\bigsqcup$ ” indicates the disjoint union) by $\xi(f):=f_{*}$. Observe that, for each $i \in\{1, \ldots, k\}$ and for each $j \in\left\{1, \ldots, n_{i}\right\}, p_{t}\left(Y_{i j}\right) \geq p_{t}(Y) \geq 2$ because $Y_{i j}$ is an algebraic subvariety of $Y$. By Theorem 1.18, we know that each set $\operatorname{Dom}\left(X, Y_{i j}\right)$ is finite. It follows that $w \operatorname{Dom}(X, Y)$ is finite also.

\subsection{Final remark}

Let $R$ be a real closed field and let $X$ and $Y$ be affine algebraic varieties over $R$. Indicate by $\mathcal{S}^{0}(X, Y)$ the set of continuous semi-algebraic maps from $X$ to $Y$ and by $\mathcal{N}(X, Y)$ the corresponding set of Nash maps. Let us define the "compact"-open topology on $\mathcal{S}^{0}(X, Y)$ as follows. For each closed and bounded semi-algebraic subset $K$ of $X$ and for each open semi-algebraic subset $U$ of $Y$, indicate by $W(K, U)$ the set of all maps $g \in \mathcal{S}^{0}(X, Y)$ such that $g(K) \subset U$. The family of finite intersections of sets of the form $W(K, U)$ is a base of the "compact"-open topology.

Let $M$ be a Nash submanifold of $R^{m}$ of dimension $d$, let $U$ be an open semialgebraic subset of $M$, let $V$ be an open semi-algebraic subset of $R^{d}$ and let $\varphi$ : 
$U \rightarrow V$ be a map. If $\varphi$ is a Nash isomorphism, then $(U, \varphi)$ is called Nash chart on $M$. Suppose now $X$ and $Y$ nonsingular. Let $r:=\operatorname{dim}(X)$ and $s:=\operatorname{dim}(Y)$. Let us define the $C^{\infty}$ "compact"-open topology on $\mathcal{N}(X, Y)$ following Chapter 2 of [14]. Let $f \in \mathcal{N}(X, Y)$, let $(U, \varphi)$ and $(V, \psi)$ be Nash charts on $X$ and on $Y$ respectively, let $K$ be a closed and bounded semi-algebraic subset of $X$ contained in $U$ such that $f(K) \subset V$, let $\varepsilon \in\{t \in R \mid t>0\}$ and let $h \in \mathbb{N}$. Define $\mathcal{N}(f ;(U, \varphi),(V, \psi), K, \varepsilon, h)$ as the set of all maps $g \in \mathcal{N}(X, Y)$ such that $g(K) \subset V$ and

$$
\left\|D_{\alpha}\left(\psi \circ g \circ \varphi^{-1}\right)(x)-D_{\alpha}\left(\psi \circ f \circ \varphi^{-1}\right)(x)\right\|_{s}<\varepsilon
$$

for each $x \in \varphi(K)$ and for each $\alpha=\left(\alpha_{1}, \ldots, \alpha_{r}\right) \in \mathbb{N}^{r}$ with $|\alpha|=\sum_{i=1}^{r} \alpha_{i} \leq h$, where $D_{\alpha}$ indicates $\partial^{|\alpha|} / \partial x_{\alpha_{1}} \cdots \partial x_{\alpha_{r}}$ and $\|v\|_{s}:=\left(\sum_{i=1}^{s} v_{i}^{2}\right)^{1 / 2}$ for each $v=$ $\left(v_{1}, \ldots, v_{s}\right) \in R^{s}$. The family of finite intersections of sets of the form $\mathcal{N}(f ;(U, \varphi),(V, \psi), K, \varepsilon, h)$ is a base of the neighborhood system of $f$ in $\mathcal{N}(X, Y)$ for the $C^{\infty}$ "compact"-open topology.

\section{References}

[1] A. Alzati and G. P. Pirola, Some remarks on the de Franchis theorem, Ann. Univ. Ferrara 36 (1990), 45-52.

[2] E. Arbarello, M. Cornalba, P. A. Griffiths and J. Harris, "Geometry of Algebraic Curves", Vol. I. Grundlehren Math. Wiss., Vol. 267, Springer-Verlag, New York, 1985.

[3] R. Benedetti and A. Tognoli, On real algebraic vector bundles, Bull. Sci. Math. 104 (1980), 89-112.

[4] J. Bochnak, M. Coste and M.-F. Roy, "Real Algebraic Geometry", Translated from the 1987 French original. Revised by the authors. Ergeb. Math. Grenzgeb. (3), Vol. 36, Springer-Verlag, Berlin, 1998.

[5] J. BOCHNAK and W. KUCHARZ, The Weierstrass approximation theorem and a characterization of the unit circle, Proc. Amer. Math. Soc. 127 (1999), 1571-1574.

[6] J. BOCHNAK and W. KUCHARZ, The Weierstrass approximation theorem for maps between real algebraic varieties, Math. Ann. 314 (1999), 601-612.

[7] J. BochnaK and W. KUCharZ, Smooth maps and real algebraic morphisms, Canad. Math. Bull. 42 (1999), 445-451.

[8] J. BOCHNAK and W. KUCHARZ, Line bundles, regular mappings and the underlying real algebraic structure of complex algebraic varieties, Math. Ann. 316 (2000), 793-817.

[9] J. BochnaK, W. KUCHARZ and R. Silhol, Morphisms, line bundles and moduli spaces in real algebraic geometry, Inst. Hautes Études Sci. Publ. Math. 86 (1997), 5-65 (1998); Erratum to: "Morphisms, line bundles and moduli spaces in real algebraic geometry" [Inst. Hautes Études Sci. Publ. Math. 86, (1997), 5-65 (1998); MR 99h:14055], Inst. Hautes Études Sci. Publ. Math. 92 (2000), 195 (2001).

[10] M. Coste and M. Shiota, Nash triviality in families of Nash manifolds, Invent. Math. 108 (1992), 349-368.

[11] M. De Franchis, Un teorema sulle involuzioni irrazionali, Rend. Circ. Mat. Palermo 36 (1913), 368.

[12] R. GHILONI, On the space of real algebraic morphisms, Atti Accad. Naz. Lincei Cl. Sci. Fis. Mat. Natur. Rend. Lincei (9) Mat. Appl. 14 (2003), 307-317 (2004). 
[13] R. Ghiloni, Rigidity and moduli space in Real Algebraic Geometry, Math. Ann. 335 (2006), 751-766.

[14] M. W. HIRSCH, "Differential Topology", Springer-Verlag, Berlin, 1976.

[15] A. HowARD and A. J. Sommese, On the theorem of de Franchis, Ann. Scuola Norm. Sup. Pisa Cl. Sci. (4) 10 (1983), 429-436.

[16] N. JoglaR-PRIETO, Rational surfaces and regular maps into the 2-dimensional sphere, Math. Z. 234 (2000), 399-405.

[17] N. Joglar-Prieto and J. KolláR, Real abelian varieties with many line bundles, Bull. London Math. Soc. 35 (2003), 79-84.

[18] N. Joglar-Prieto and F. Mangolte, Real algebraic morphisms and Del Pezzo surfaces of degree 2, J. Algebraic Geom. 13 (2004), 269-285.

[19] E. KANI, Bounds on the number of non-rational subfields of a function field, Invent. Math. 85 (1986), 185-198.

[20] W. KUCHARZ, Algebraic morphisms into rational real algebraic surfaces, J. Algebraic Geom. 8 (1999), 569-579.

[21] W. KUCHARZ and K. RUSEK, Approximation of smooth maps by real algebraic morphisms, Canad. Math. Bull. 40 (1997), 456-463.

[22] F. MAngolte, Real algebraic morphisms on 2-dimensional conic bundles, Adv. Geom., to appear.

[23] H. MARTEns, Observations on morphisms of closed Riemann surfaces, Bull. London Math. Soc. 10 (1978), 209-212.

[24] J. C. NARANJo and G. P. Pirola, Bounds of the number of rational maps between varieties of general type, to appear.

[25] J.-P. SERRE, Faisceaux algébriques cohérents, (French), Ann. Math. 61 (1955), 197-278.

[26] F. SEVERI, Sugli integrali abeliani riducibili, Rend. Mat. Acc. Lincei Ser. V, 23 (1914), 581-587.

[27] M. SнiотA, "Nash Manifolds", Lecture Notes in Mathematics, Vol. 1269, Springer-Verlag, Berlin, 1987.

[28] M. TAnABE, A bound for the theorem of de Franchis, Proc. Amer. Math. Soc. 127 (1999), $2289-2295$.

Department of Mathematics University of Trento

Via Sommarive, 14 38050 Povo (TN), Italy ghiloni@science.unitn.it 